East African Medical Journal Vol. 86 No. 1 January 2009

THYROID DISEASES IN PREGNANCY: A REVIEW

A.W.O. Olatinwo, MBBS, FWACS, MBA, O.O. Fakeye, MBBS, FACOG and S.A.I. Lawal, MBBS, Senior Registrar, Department of Obstetrics and Gynaecology, University of Ilorin Teaching Hospital, Ilorin, Nigeria

Request for reprints to: Dr. A.W.O. Olatinwo, Department of Obstetrics and Gynaecology, University of Ilorin Teaching Hospital, Maternity Hospital Wing, Ilorin, Nigeria

\title{
THYROID DISEASES IN PREGNANCY: A REVIEW
}

\author{
A.W.O. OLATINWO, O.O. FAKEYE and S.A. I. LAWAL
}

\begin{abstract}
Objective: To provide an overview of the pathophysiology of thyroid diseases in pregnancy and the various treatment methods currently available in its management.

Data source: Review of literature was affected through index medicus search of major published journals and books.

Data selection: Published data on thyroid diseases on women in the reproductive age group over the last four decades were utilised.

Data extraction: Abstracts and full text of selected manuscripts were read and analysed to determine their contribution to this article.

Data synthesis: All relevant articles were reviewed and contributions extracted.

Conclusion: Thyroid diseases are the second most common endocrinopathies in pregnancy. Numerous hormonal changes and metabolic demands occur in pregnancy resulting in complex changes in maternal thyroid function. Thyroid diseases involves a multidisciplinary team approach to achieve good metabolic control and excellent obstetric care in order to ensure normal maternal and perinatal outcome.
\end{abstract}

\section{INTRODUCTION}

Thyroid disorders are the second most common endocrinopathies found in pregnancy (1). Significant thyroid disease complicates about $0.8 \%$ of all pregnancies approximately $0.2 \%$ hyperthyroid and $0.6 \%$ hypothyroid (2). Disease of the thyroid are generally much more prevalent in females than in the males and with marked preference for women in the reproductive age group, of which 5 - 15\% may be affected (3). The prevalence of thyroid disease in pregnancy is however less than expected for the age-related population because thyroid diseases significantly affect fertility rate and autoimmune thyroid disease tend to remit during pregnancy $(2,4)$.

Numerous hormonal changes and metabolic demands occur in pregnancy, resulting in complex changes in maternal thyroid function, yet normal pregnancy is considered to be a euthyroid state (5). Pregnancy itself does not cause abnormal thyroid function, but rather, hypothyroidism and hyperthyroidism may be found in pregnant and non-pregnant women alike.

Thyroid gland changes in pregnancy: The thyroid gland enlarges in pregnancy due to increased vascularity and cellular hyperplasia. Maternal thyroid function is modulated by three independent but interrelated factors (5).

(i) Anincrease in thyroxin-binding globulin(TBG) during the first trimester, resulting in increased binding of thyroxin.

(ii) An increase in HCG (TRH-like activity) concentration that stimulate the thyroid gland.

(iii) Significant increase in urinary iodine excretion resulting in a fall in plasma iodine concentration and;

In the aggregate, these factors may be responsible for the increased thyroid demand or thyroid 'strain' observed during pregnancy. The end result is a relatively elevated thyroid hormone in early pregnancy and this may be necessary for normal foetal development, especially or the foetal nervous system (6).

Pregnancy in sub-clinical hypothyroid disorders: Hypothyroidismin pregnancy is rare whilesubclinical hypothyroidism is more commonly encountered (7). The clinical diagnosis of sub clinical hypothyroidism is based on the chemical analysis indicating a normal free thyroxin level und a slightly elevated thyrotrophic level. The aetiologies of hypothyroidism include 
iodine deficiency, autoimmune thyroiditis, post thyroid ablation therapy and post thyroidectomy.

The spectrum of women with overt or sub clinical hypothyroid in pregnancy include women who discontinue thyroid hormone therapy, those on therapy requiring larger doses, hyperthyroid patients on excessive amount of antithyroid drugs and some patients on lithium or amiodarone therapy $(9,10)$. Patients with sub clinical disease are usually asymptomatic and may get pregnant and when they do, the symptoms of mild hypothyroidism mimic those of normal pregnancy, making diagnosis difficult. These symptoms include lethargy, constipation, weight gain, muscle cramping, carpel tunnel syndrome, dry skin and hair and a deeper voice.

Maternal morbidity associated with hypothyroidism includes microcytic anaemia pre eclampsia, placental abruption, post partum haemorrhage, cardiac dysfunction and pregnancy wastage. Foetal or neonatal complications include prematurely, low birth weight, congenital anomalies, stillbirths and poor neuropsychological development (1).

Maternal replacement therapy and close monitoring is indicated in sub-clinical disease to normalise maternal thyrotrophin levels. When the foetal thyroid is normal and there is no deficiency, maternal hypothyroidism may not adversely affect subsequent mental development of the offspring (10). With only mild elevation of TSH as seen in sub clinical hypothyroidism, the foetus is at less risk from mild maternal thyroid failure and TSH does not cross the placenta.

The effects of thyroid disease on the foetus: Studies in the last two decades have shown an important role of maternal thyroid hormones in embryogenesis (11). The foetal thyroid only begins to produce significant amount of thyroid hormones by 18 to 2 weeks gestation. Prior to that time, the foetus is completely dependent upon transplacental transfer of maternal thyroid hormones. Adverse effect on the intellectual development may occur in children of mothers with mild thyroid deficiency in the first 20 weeks of gestation (12).

Foetal complications of maternal hyperthyroidism include spontaneous abortion, premature labour, stillbirths, low birth weight and foetal/neonatal hyperthyroidism. Foetal thyrotoxicosis may result from transplacental thyroid stimulating antibodies, giving rise to widespread foetal autoimmune reaction with lymphatic hypertrophy, thrombocytopenia, foetal goiter and exophthalmos (14).

Foetal or neonatal hypothyroidism may follow maternal administered thioamides which block thyroid hormone biosynthesis in the foetus. Transplacental passage of maternal thyrotropin- receptor blocking antibodies may cause foetal hypothyroidism in women with autoimmune thyroid diseases. Maternal iodine deficiency also results in foetal/neonatal hypothyroidism with or without goiter in the foetus (star gazing foetus). Other foetal complications of maternal hypothyroidism include spontaneous abortion, low birth weight, foetal death and lower intelligent Quotient (13). Treatments include maternal thyroxine therapy as well as use of intraamniotic thyroxine injection. In mothers being managed on antithyroid medication, the smallest dose that maintains maternal free hormones and TSH within the normal range should be used in order to avoid foetal hypothyroidism.

Post-partum thyroiditis: This condition presents as hypothyroidism (40\%), hyperthyroidism $(30 \%)$ or hyperthyroidism followed by hypothyroidism (12). It's a transient autoimmune thyroidities found in 5$10 \%$ of women during the first year after childbirth (15). The propensity for thyroiditis antedates pregnancy and is directly related to the increasing level of thyroid auto antibodies. Thyroid biopsy shows lymphocytic thyroiditis. Risk factors include previous thyroid dysfunction, hereditary thyroid or other autoimmune diseases and type 1 diabetes mellitus (16).

Two clinical phases of post partum thyroiditis are known (4). In the first four months post delivery, transient destruction-induced thyrotoxicosis from excessive release of hormones from glandular disruption occurs in about $4 \%$ of women. It is of abrupt onset and is characterised by small painless goiter, fatigue and palpitation. Antithyroid drugs are ineffective but Beta adrenergic-blockers may be given. Two-third of patients return to euthyroid state while the remaining develops transient or permanent hypothyroidism.

Between four to eight months post partum 2-5\% of women develop hypothyroidism groin thyroiditis. A third of these would have gone through the thyrotoxicosis phase. Thyroxine replacement may be given for 6-12 months, but some may have developed permanent hypothyroidism.

In clinical practice however, thyroiditis is infrequently diagnosed because it ty pically develops months after delivery and has nonspecific symptoms such as depression and memory impairment which are easily attributed to other causes.

Effects of antithyroid drugs in pregnancy: Antithyroid drug regimen is the appropriate treatment for most women with hyperthyroidism in pregnancy. Radioactive iodine is contraindicated in pregnancy while surgery is reserved for those with significant drug intolerance or extremely poor compliance.

The standard therapy for hyperthyroidism is the use of antithyroid drugs with the aim of reducing the 
circulating free $\mathrm{T}^{4}$ concentration in to the high normal or just elevated range. Close monitoring with frequent measurement of TSH and T ${ }^{4}$ must be carried out twice weekly at the beginning of treatment and every 2-4 weeks when euthyroidism is achieved (7).

The commonly used antithyroid drugs are propylthiouracil and methimazole; both are thiamine drugs which act by blocking hormone synthesis at the start of organification and iodotyrosine coupling. Both drugs are effective in controlling symptoms and no one is superior to the other in terms of efficacy, (17) though aplasia cutis was reported in association with methimazole (18). Side effects of antithyroid drugs occur in 3-5\% treated patients (19). The most common complications of both drugs are pruritus and skin rash. Other less common complications are migratory polyarthritis, a lupus like syndrome and cholestatic jaundice. Agranulocytosis is a serious but unusual complication which necessitates discontinuation of the drugs. Of clinical significance is the effect of antithyroid drugs on the foetus. Excessive amount of antithyroid drugs are associated with foetal hypothyroidism and goitre. Because of the unpredictable transplacental passage of thyroid hormone, supplementation with either $\mathrm{T}^{3}$ or $\mathrm{T}^{4}$ provides a false sense of security, making the mother euthyroid while continuing foetal exposure to high doses of antithyroid drugs than would be necessary without thyroid hormone supplementation. Use of adjunctive therapy with stapleiodine is also not justified in pregnancy because it crosses the placenta easily and may be responsible for development of large foetal goitre, hypothyroidism and foetal death (20). Patients with small goiters or short duration of symptomsand those on minimal amount of antithyroid medication may be able to discontinue antithyroid medications by 34 weeks and beyond (7).

Propanolol and other adrenergic-blocking drugs have been associated with placental insufficiency, intrauterine growth (21) retardation, excessive uterine irritability and foetal bradycardia. Their use should therefore be restricted to severe cases of hyperthyroidism.

Thyroid storm in pregnancy: Thyroid storm is a lifethreatening condition with clinical diagnosis based on severe signs of thyrotoxisis; it is characterised by significant hyperpyrexia $\left(103^{\circ} \mathrm{F}\right)$, neuropsychiatry's symptoms, nausea and vomiting. It is rare in pregnancy but could be precipitated by delivery, acuteillness, infection or surgery (22). It requires early recognition and aggressive therapy in an intensive care unit. Management includes supportive therapy with fluids, correction of electrolyte abnormalities, oxygen therapy as needed and control of hyperpyrexia with acetaminophen. Antibiotics could also be given in case of infection.
Beta-blockers which acts by suppressing adrenergic output and block peripheral conversion of $\mathrm{T}^{4}$ to $\mathrm{T}^{3}$ are also useful. It is given as $60-80 \mathrm{mg}$ every 4 four hours orally or $1 \mathrm{mg} / \mathrm{min}$ intravenously. Methimazole $30 \mathrm{mg}$ or propylthiouracil $300 \mathrm{mg}$ every 6 hours is given orally or via NG tube. They block the synthesis of thyroid hormone in a few hours. One hour after the administration of thioamides, iodine is administered in form of lugol's solution, 10 drops 8 hourly or if available, sodium iodide $1 \mathrm{~g}$ intravenously every 12 hours. Intravenous dexamethazole $2 \mathrm{mg} 6$ hourly for 4 doses also help to further block peripheral conversion $\mathrm{T}^{4}$ to $\mathrm{T}^{3}$.

Pregnancy post-thyroidectomy: Different clinical situations may be met when counselling women with current or past thyroid diseases contemplating pregnancy. Hyperthyroid women under treatment may be taking antithyroid drug therapy and the potential sideeffects of antithyroid drugs on the foetus should be discussed. Those who have had radioactive ablation need to wait six months after the therapeutic dose is administered before contemplating pregnancy while those who had had surgery still needs to be euthyroid at the time of conception. Previous ablation treatment for Grave's disease or thyroid carcinoma requires adjustment in the dose of thyroid replacement therapy soon after conception (23) and the risk of foetal hyperthyroidism despite maternal euthyroidism still exist in mothers with high titres of TSI following ablative therapy.

\section{CONCLUSION}

Thyroid disorders in pregnancy can be adequately tackled with multidisciplinary team approach for successful outcomes as it's with diabetes mellitus today. It is imperative to advise women with chronic thy roid diseases to plan their pregnancies and contact their health care professional as soon as the diagnosis of pregnancy is made. Good metabolic control coupled with excellent obstetric care can ensure normal maternal and perinatal outcome.

\section{REFERENCES}

1. Nguyen, P.H. Autoimmune Thyroid Disease and Pregnancy in Emedicine World medical library. Editors: Ronald L., Francisco T., Carle V.S. Fedrick B.G Lee P.S. File: / / F autoimmune as in preg htm 2004: 1-20.

2. Terry, F.D. and Rhoda, H.C. Thyroid disease in pregnancy and post partum period. In: Cherry and Merkatz's complications of pregnancy 5th edition Wayne, R.C.Shelden H.C.Ivcuin R.M. (ed) Lippincott, Williams and Wilkins (pub) 2000. wwww.isilo. Chapter 22: 29-68. 
3. Tunbridge, W.M.G., Evered, D.C, Hall, R. et al. The spectrum of thyroid disease in a community. Clin. Endocrinol. 1977; 164: 1439-1447.

4. Cunningham, F.G., Leveno, K.J., Bloom, et al. (ed). Thyroid and other endocrine disorder. In: William's obstetric. McGramr - Hill (pub )2005; pp 1190-1198.

5. Gerard, N.B. and Lauren, H.G. Thyroid disease during pregnancy file/ pregnancy. htm. 2002; 1-9.

6. Iskaros, J., Pickard, M., Evans, I., et al. Thyroid hormone receptor geneexpression in the first trimester human fetus brain. J. Clin. Endocrinol. Metab. 2000; 85: 2620.

7. Jorge, H.M. Endocrine disease in pregnancy. In: Gabbe Obstetrics - normal and problem pregnancies 4th Edition chapter 33. GabbeS.G., Niebyi J.R.Simpson J.I. (ed) Churchill Livingstone (pub) 2002; 1117-1168.

8. Harjai, K.L and Licata, A.A. Effects of amiodarone on thyroid function. Ann. Intern. Med. 1997; 126:63.

9. Wilson, R., Mckillop, K.H., Crocket, G.T., et al. The effect of lithum therapy on parameters thought to be involved in the development of autoimmune thyroid disease. Clin. Endocrinol. 1991; 34: 357.

10. Liu, H., Momotani, N. and Yoshimura J. Maternal hypothyroidism during pregnancy and intellectual development of the progeny. Arch. Intern. Med. 1994; 154: 785-787.

11. Porterfield, S.P. and Hendrich C.E. The role of thyroid hormone in prenatal and neonatal neurological development: current perspectives. Endocr. Rev. 1993; 14: 94.

12. Haddow, J.E., Palmomaki, G.E., Allan, W.C., et al. Maternal thyroid deficiency during pregnancy and subsequent neuropsychological development or the child. N. Engl. J. Med. 1999; 341: 549.
13. Sandra Lowe: Thyroid disease and pregnancy. J. Obs. Gynae. 2006; 8: 26-27.

14. J.D. Seffah. Thyroid diseases in pregnancy In: Comprehensive obstetrics in the tropics E.Y Kwanwukume E.E. Emuveyan (cd) 2002; 317-320.

15. Aminu, N., Tada, H., Hidaka, Y., et al. Post partum thyroid syndrome. Endocr. J. 2000; 47:645.

16. Alvarez-marfany, M., Roman, S. H., Drexler, A. J., et al . Long term prospective study of post partum thyroid dysfunctionin women withinsulin dependent diabetes mellitus J. Clin. Endocrinol. Metab. 1994; 79: 10-15.

17. Mortimer, R.H., Cannal, G.R., Addsion, R.S., et al. Methimazole and propylthiouracil equally cross the perfused human term placental lobule. J. Clin. Endocrinol. Metab. 1997; 82: 3099.

18. Mandel, S.K., Brent, G.A. and Larsen, P.R. Review of antithyroid drug use during pregnancy and report of a case of aplasia cutis. Thyroid. 1994: 4: 129.

19. Franklyn, J. A. The management of hyperthyroidism N. Engl. J. Med. 1994; 330: 1731-1735.

20. Galina, M.P.,Artnet, M.I. and Einjorn, A. Iodides during pregnancy N. Engl. J. Med. 1962; 267: 1124-1127.

21. Pruyn, S.C., Phelan, J.P. and Buchanan G.C. Long term propranolol therapy in pregnancy: maternal and fetal outcome. Assoc. Med. J. Obstet Gynaecol. 1979; 135:485-489.

22. Dipika, D., Wayne, H., Darla, B. H. and John, C.M. General medical disorders during pregnancy. In: Current obstetric and gyneacologic diagnosis and treatment De herney A.H Nathan L. (ed) $9^{\text {th }}$ edition. McGraw - Hill (Pub) 2003. pp 424-450.

23. Kaplan, M.M. Management of women on thyroxine therapy during pregnancy. Endocr. Pract. 1996; 2: 281. 